\title{
Enantioselective conversions by bacterial quinoprotein alcohol dehydrogenases
}

\author{
J.A. Duine, J.A. Jongejan and A. Geerlof \\ Department of Microbiology \& Enzymology, Delft University of \\ Technology, Julianalaan 67, 2628 BC Delft, The Netherlands.
}

Bacteria contain alcohol dehydrogenases (ADH'S) which either occur in the cytosol and which interact with the NAD(P) pool or are directly coupled to the respiratory chain and can be assayed in vitro with artificial electron acceptors, i.e. by decoloration of dyes. During the past ten years, several of these dye-linked ADH's have been characterized and shown to be quinoproteins, i.e. enzymes with a quinone cofactor (in most cases this is pyrroloquinoline quinone, $P Q Q)$. These enzymes were investigated here for kinetic resolution of the $c$-synthons solketal (2,2-dimethyl-4-(hydroxymethyl)-1,3-diox3ilane) and glycidol (2,3-epoxy-1-propanol). It appeared that quinohaemoprotein ADH's are excellently suited for this -ince this type of enzymes has adequate substrate diversity, catalytic activity and resolving power, in vitro as well as in vivo.

\section{INTRODUCTION}

Certain derivatives of glycerol are attractive building blocks for the synthesis of pharmaceuticals and other specialties [1, 2]. This is related to the possibility to introduce chirality at an early stage in synthesis routes by using the enantiomerically pure forms of these $c$-synthons. Interesting examples in this respect are glycidol $(2,3-$-epoxy-1-propanol) and solketal (2,2dimethyl-4-(hydroxymethyl)-1,3-dioxolane), the structures shown on the left side. Chemical synthesis routes have been developed<smiles>C[C@H]1OC[C@H](CO)O1</smiles>

S-Sotketal<smiles>OC[C@H]1CO1</smiles>

S-Glyeidal for the enantiomers, but application of enzymatic kinetic resolution is another approach. This has already been applied for production of R-glycidyl butyrate (available from a number of companies) in three steps: first the ester racemate is prepared by chemical synthesis from commercially available, relatively cheap racemic glycidol; the ester racemate is hydrolyzed with pig pancreas lipase (having a preference for the $S$-ester) in the presence of water for a certain time; R-glycidyl butyrate is removed from the water, glycidol, and butyrate. However, this process has an inherent drawback. Due to the kinetic behaviour of pancreas lipase (as well as other serine hydrolases), substantial back reaction occurs with the R-glycidol formed, competing with $\mathrm{H} O$ for the acylated enzyme form, when most of the s-glycidyl bủtyrate is hydrolyzed (at approx. an eextent of conversion of $60 \%$ [3]). This phenomenon is prohibitive to reach higher e.e. values than $98 \%$ when high concentrations of the ester are used and only one batch reaction is applied, 
conditions crucial for economic feasibility. Therefore, it seemed interesting to follow another approach, i.e. to circumvent esterification and apply direct kinetic resolution to the racemic $C$-alcohols via alcohol-oxidizing enzymes (alcohol oxidoreductasẻs).

Alcohol oxidoreductases are rubricated by the Enzyme Nomenclature Commission under the heading EC 1.1.-.-. This group can be subdivided (reflected by the third digit) into alcohol oxidases (EC 1.1.3.-), NAD ( P) -dependent alcohol dehydrogenases

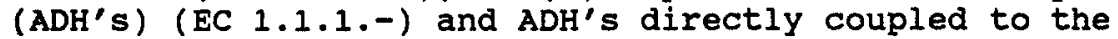
respiratory chain and which can be assayed in vitro through decoloration of artificial electron acceptors (dye-linked ADH's) (EC 1.1.99.-). Of these subgroups, only NAD-dependent ADH's have been investigated with respect to enantioselectivity, most of this work being carried out with ADH from horse liver and bakers yeast [4]. However, due to the unfavorable redox potential of $\mathrm{NAD} / \mathrm{NADH}$ and the frequently occurring product inhibition of these enzymes, investigations have been mainly restricted to the reverse reaction, the conversion of prochiral aldehydes or ketones into the corresponding alcohol enantiomer(s). Screening of the subgroups has been carried out by us for solketal and glycidol [5]. It appeared that alcohol oxidases and NAD(P)dependent $A D H^{\prime}$ 's had inferior qualities with respect to selectivity. The work presented here focuses on enantioselectivity towards the aforementioned $c$-synthons of a novel type of dye-linked ADH's which have pyrroloquinoline quinone (PQQ; 2,7,9-tricarboxy-1H-pyrrolo[ $2,3 f$ ] quinoline-4,5dione) as their cofactor, the structure of the redox forms depicted below.<smiles></smiles>

poo<smiles>O=C(O)c1cc(C(=O)O)c2c(n1)c(O)c(O)c1cc(C(=O)O)[nH]c12</smiles>

POOH.<smiles>O=C(O)c1cc(C(=O)O)c2c(n1)c(O)c(O)c1cc(C(=O)O)[nH]c12</smiles>

POOH

These quinoprotein ADH's occur in microbes with an extremely high capacity to oxidize alcohols, well known examples being found in the Acetic acid bacteria. Since some of these enzymes appeared to be qualified in vitro, the organism from which they were derived were inspected for the same qualities as well as for other properties relevant for application.

\section{OUINOPROTEIN ALCOHOL DEHYDROGENASES}

PQQ was originally detected in methanol dehydrogenase (MDH), an enzyme reponsible for conversion of methanol into formaldehyde in C-compounds (methane, methanol)-converting, Gram-negative bacteria (see a recent review [6] on quinoproteins and the quinone cofactors). A similar enzyme appears to be present in bacteria (especially Pseudomonads) growing on alcohols higher than methanol, given the arbitrary name ethanol dehydrogenase (EDH). Both enzymes are tetramers containing 2 $P Q Q^{\prime} s$ and 2 Ca ions. The redox couple of $P Q Q / P Q Q H$ is rather high $(+100 \mathrm{mV}$ at $\mathrm{pH} 7)$ when compared with that of ${ }^{2} \mathrm{NAD} / \mathrm{NADH}$. Thus alcohol oxidation by the oxidized form of the enzyme gives no problem in a thermodynamic sense. Most probably related to the high redox potential of these quinoproteins, reoxidation in vivo of the reduced enzyme form occurs at the level of cytochrome $c$. 
Quinohaemoprotein ethanol dehydrogenase (QH-EDH) was originally discovered in Comamonas testosteroni [7]. The enzyme is produced in the apo-form, i.e. the organism does not produce $P Q Q$, implying that growth on alcohols must proceed via another ADH (low activities of NAD-dependent ADH are found indeed in cell free extract). Reconstitution (in vivo and in vitro) is easily achieved with $\mathrm{PQQ}$ and Ca salts. Thus holo-enzyme contains $P Q Q$ as well as a haem $c$ group. Acetobacter and Gluconobacter species contain a somewhat similar enzyme [8]. However, in contrast to the $c$. testosteroni enzyme, it contains a separate haem $c$-containing subunit, reason why the enzymes are distinguishable and indicating them here as type $I$ and type II, respectively. It has been shown [9] that bacteria growing on polyethyleneglycol (PEG) contain a PEG-dehydrogenase which could be a quinoprotein. Although it has been suggested that PQQ is present, the identity of the cofactor has yet to be established.

\section{METHODS TO DETERMINE ENZYME SELECTIVITY}

The enantioselectivity of an enzyme for a certain racemic substrate is expressed by the enantiomeric ratio, E, which is defined in the following way:

$$
E=\frac{\left(V_{1} / R_{M}\right)}{\left(V_{m} / K_{M}\right)} \text {, in which the } R \text { enantiomer is preferred by the }
$$

The presently existing methods will be discussed below.

\section{From the kinetic parameters}

As is in the name, dye-linked alcohol dehydrogenases can be assayed with artificial electron acceptors. Since the redox potentials of these compounds as well as of the cofactor are rather high, the oxidations are practically irreversible, as illustrated by the high value of the equilibrium constant ( 2 . $10^{2}$, calculated for oxidation of ethanol to acetaldehyde with ferricyanide via the enzyme (at $1 \mathrm{mM}, 25^{\circ} \mathrm{C}$, pH 7). Thus all methods for $\mathrm{E}$ determination presented here are applicable.

Since quinoprotein ADH's also oxidize aldehydes, the kinetic picture is complicated by the fact that substrate and product compete for the same active site. However, by analyzing the the progression curves for dye decoloration and acid formation (with a pH-stat), the kinetic parameters could be resolved for alcohol as well as aldehyde oxidation (J.L.I. Rakels, A. Geerlof, A.J.J. Straathof, J.A. Jongejan, J.J. Heijnen and J.A. Duine, unpublished results). For the approach based on initial rate measurements (methods $a$ and $b$, see below), it was assumed that aldehyde concentrations are still so low that they would not interfere with the alcohol conversion.

a) When the pure enantiomers of the substrate are available, determination of the kinetic parameters for each of them by initial rate measurements enables calculation of the $E$ value according to the definition given above. Inherent to the approach, the method will detect the occurrance of substrate inhibition. However, if the less preferred enantiomer is contaminated with a slight amount of the preferred enantiomer (or another substrate), large errors can be expected. Moreover, neither product inhibition nor any other interfering events (e.g. enzyme inactivation, back reaction due to product accumilation) occurring during the bioconversion process will be detected with this method. 
b) When the concentration of the racemic substrate is kept constant but the ratio of the enantiomers is varied, a rather simple relationship can be derived for this situation [10]:

$$
E=\frac{v_{\mathbf{s}}\left(v_{-x}-v_{R}\right)}{v_{R}\left(v_{s}-v_{x}\right)} \cdot \frac{1-x}{x}
$$

Thus when the pure enantiomers are not available but samples can be prepared with varying ratio of the enantiomers (and these ratios can be calculated or determined by an analytical procedure), E can be estimated from initial rates measured for at least three $x$-values (molar fraction of the s-enantiomer). The disadvantages mentioned for method a also apply here.

\section{Erom progression curves}

Integration of the equation defining $\mathbf{E}$ gives the following relationship between $E$, the enantiomeric excess of the remaining substrate (ee ${ }_{s}$ ) and the extent of conversion ( $\$$ ) [11]:

$$
E=\frac{\ln [(1-e e) \cdot(1-\S)]}{\ln \left[\left(1+e e_{\mathrm{s}}\right) \cdot(1-\S)\right]}
$$

This equation forms the base of method $c$ (see below).

c) By assuming that the type of kinetics of the enzyme allow the use of the equation and that it is valid from start to end of the bioconversion, $E$ can be determined from a single point in the progression curve for which ee and $\S$ are determined. Reversibly, by examining several points, the constancy of $E$ at these points gives an indication whether any irregularities occur during the course of the reaction. Moreover, with this a more reliable $\mathrm{E}$ value can be estimated since a curve fitting procedure can be applied to the data points lying in the range where they can be most accurately determined.

Enantiomeric excess and extent of conversion. Starting with a racemic mixture and converting this in a way sothat the remaining substrate or the product formed is enantiomerically pure, the enantiomeric excess of the product wanted varies from 0 to $100 \%$. Thus reliable and accurate methods should be available to determine the ratio of the enantiomers in this range. Originally, we adapted an existing procedure to estimate the e.e. values of glycidyl butyrate by converting the enantiomers into diasteromers and separating these with HPLC on a reversed phase column [12]. Subsequently, it appeared that other glycidyl esters and glycidol itself could be analyzed in the same way and by tosylating solketal, a modified procedure could be developed too for this compound. The method appeared to be very suited since no racemization or kinetic enrichment occurred. During the course of our studies, capillary GC columns with chiral stationary phases became availabe. It appeared that derivatization could be circumvented and direct injection (to avoid deterioration of the column by water, buffer salts and protein, it is advizable to extract the analyte into an organic solvent) gave satisfactory results, as is illustrated in Fig. 1 for the baseline-separation (between 16 and $18 \mathrm{~min}$.) of the solketal enantiomers, the results showing that an accuracy of \pm $0.1 \%$ in enantiomeric purity determination can be easily reached [13]. 


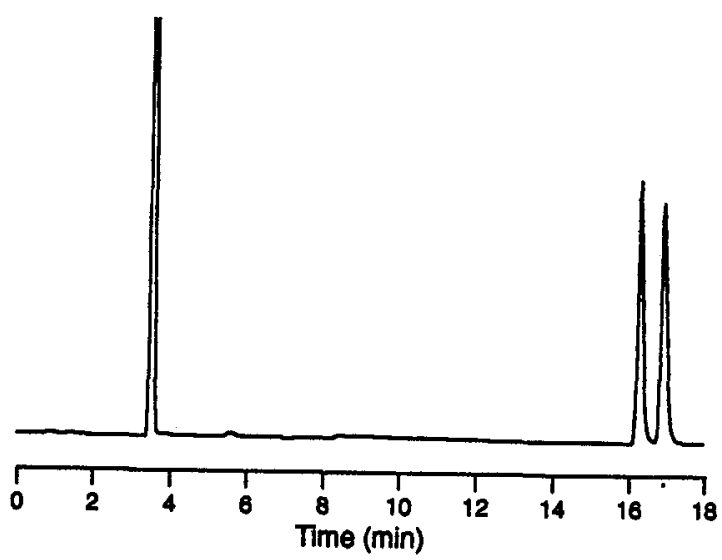

Fig. 1. GC of racemic solketal on a capillary column with chiral stationary phase.

\section{SELECTIVITY OF THE ENZYMES}

Table 1 shows the results obtained with quinoprotein ADH's [5], either in purified form or present in cell free extract where it is assumed to be the dominating $\mathrm{ADH}$. from the preferences and the $\mathrm{E}$ values determined, it appears that $a$ quinoprotein exists for nearly each $C$-synthon enantiomer. Although it concerns unnatural substrătes, for certain enzymes the rates are similar to that for ethanol [5].

TABLE 1. Selectivity of quinoprotein alcohol dehydrogenases for solketal and glycidol.

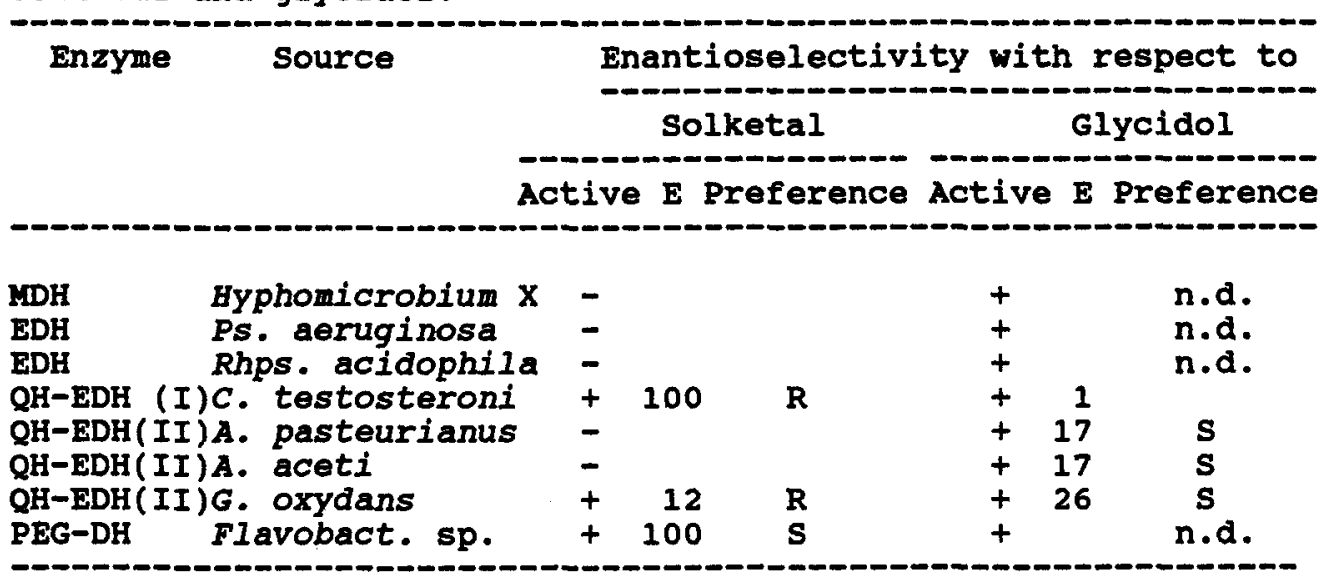

\section{PERFORMANCE OF THE BACTERIA}

Table 2 shows the results for the bacteria of which the enzymes in Table 1 were derived. For some cases, the performance of the bacterium is less than of the enzyme, as illustrated in Fig. 2. Several explanations can be given for this discrepancy; although the quinoproteins considered here are periplasmic enzymes, $i, e$, are at the outside of the cytoplasmic membrane, the hydrophobicity of this space may cause substrate limitation (solketal and glycidol are water-soluble compounds) so that substrate limitation occurs, leading to a significantly lower 
apparent $E$ value for the bacterium compared to the genuine $E$ value of the enzyme in vitro; the different micro-environments may induce differences in enantioselectivity of the enzyme; the bacterium may contain additional ADH's. At first sight, the latter possibility seems unlikely since the assays showed other enzymes to be absent or to have low activity. However, Rhps. acidophila has excellent selectivity and activity for solketal, but cell free extracts (with EDH) showed no activity. In addition, when certain organisms claimed in a patent [14] to oxidize s-solketal with high selectivity, neither quinoproteins nor PQQ was detected. Preliminary results show that one of these organisms, Rhodococcus erythropolis, contains a nicotinoprotein $A D H, i . e$. an enzyme with firmly bound NAD which has to be assayed with uncommon artificial electron acceptors (nitrosoanilines) since NADH formed in the enzyme does not interact with external NAD [15], shows the preference exhibited by the bacterium (unpublished results). Thus the presence of this type (or others) of enzyme may have been overlooked in the bacteria investigated here and reponsible for the deviations observed.

TABLE 2. Enantioselectivity of quinoprotein alcohol dehydrogenase-containing bacteria.

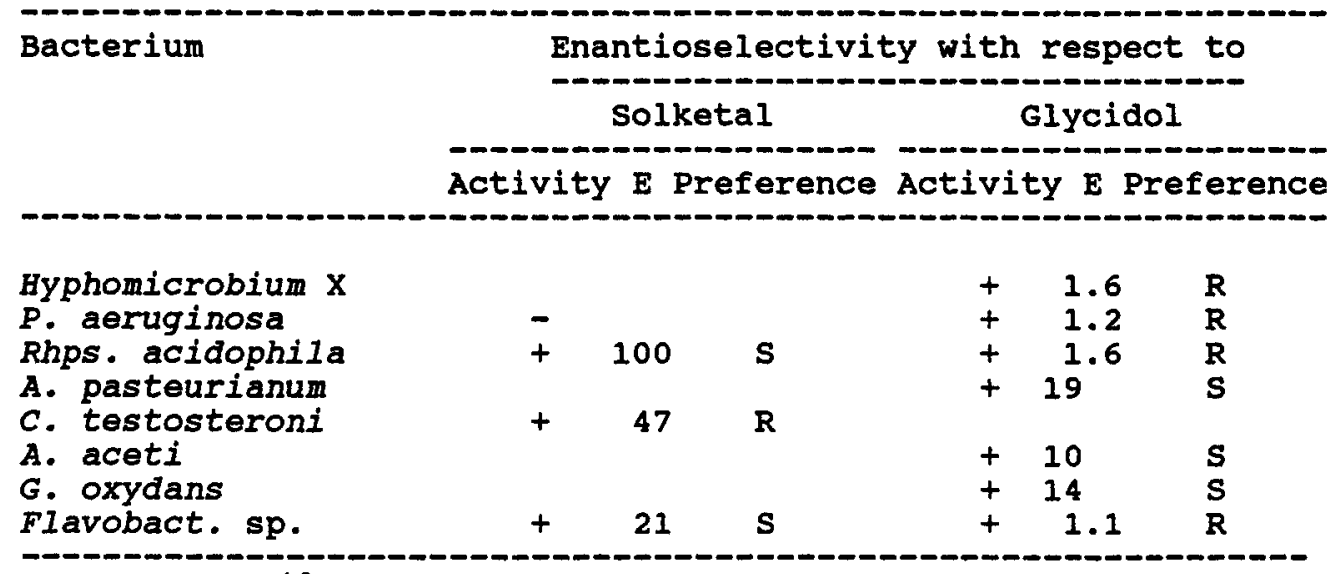

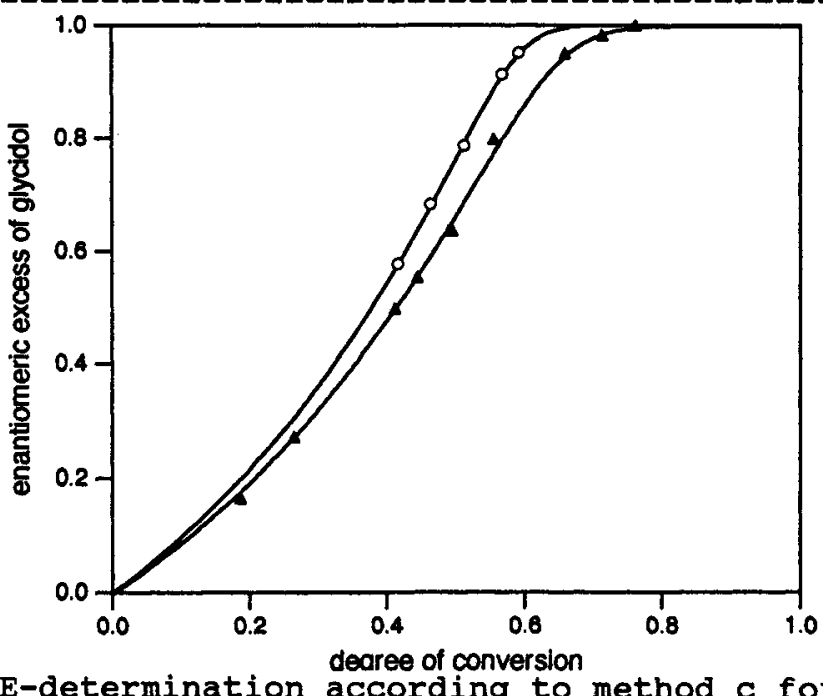

Fig. 2. E-determination according to method c for QH-EDH (type II) from Acetobacter aceti (O--0) and for the corresponding organism $(4--4)$. 
other parameters in biokinetic resolution with the bacteria Production of the biocatalyst may need the presence of the substrate to be converted in order to induce high levels of the enzyme. As shown in Fig. 3 the presence of solketal leads to a decrease in growth rate of $c$. testosteroni already at rather low solketal concentrations.

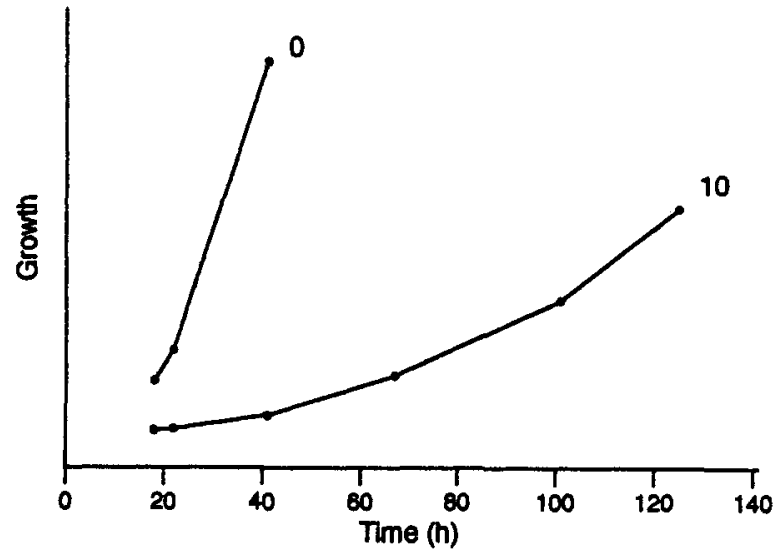

Fig. 3. Growth of comamonas testosteroni on ethanol in the absence (O) and in the presence $(10 \mathrm{~g} / \mathrm{L})$ of racemic solketal. However, induction of QH-EDH occurs at high level at growth on ethanol [7] so that the biocatalyst can be prepared in the absence of solketal. Since low concentrations of substrate are economically unattractive, it also seemed interesting to test the respiratory capacity of the cells at varying concentrations of solketal. As shown in Fig. 4, a substantial decrease was observed at concentrations higher than $0.5 \mathrm{M}$. Since this effect was irreversible, most probably this is caused by membrane damage. Further studies are in progress in order to try to circumvent these problems.

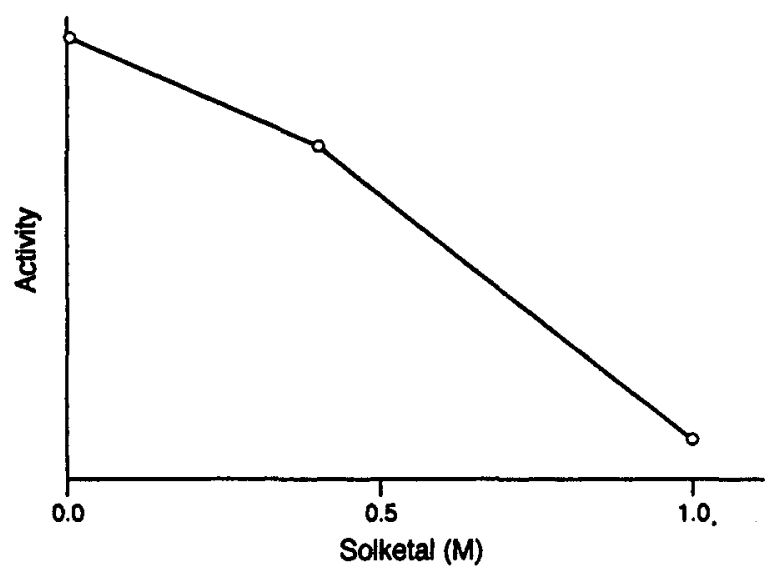

Fig. 4. Oxidative capacity of Comamonas testosteroni with varying concentrations of racemic solketal. 


\section{REFERENCES}

1. J.M. Klunder, S.Y. Ko and K.B. Sharpless, J. Org. Chem. 51 , 3710-3712 (1986).

2. U. Peters, W. Bankova and P. Welzel, Tetrahedron 43, 38033816 (1987).

3. J.B.A. van Tol, A. Geerlof, J.A. Jongejan and J.A. Duine, Ann. N. Y. Acad. Sci, 672, 462-470 (1992).

4. G.I. Lemiere, J.A. Lepoivre and F.C. Alderweireldt, Bioorg, Chem. 16, 165-174 (1988).

5. A. Geerlof, J.A. Jongejan and J.A. Duine, submitted.

6. J.A. Duine, Eur. J. Biochem. 200, 271-284 (1991).

7. B.W. Groen, M.A.G. van Kleef and J.A. Duine, Biochem. J, 234, 611-615 (1986).

8. O. Adachi, E. Shinagawa, K. Matsushita and M. Ameyama, Agric. Biel. Chem. 46, 2859-2863 (1982).

9. F. Kawai, H. Yamada, M. Ameyama, E. Shinagawa, K. Matsushita and 0 . Adachi, Agric. Biol. Chem, 49, 1071-1076 (1085).

10. J.A. Jongejan, J.B.A. van Tol, A. Geerlof and J.A. Duine, Recl. Trav. Chim. Pays-Bas 110, 247-254 (1991).

11. C.-S Chen, Y. Fujimoto, G. Girdaukas and C.J: Sih, I. Am. Chem. Soc. 104, 7294-7299 (1982).

12. M. Chr. Philippi, J.A. Jongejan and J.A. Duine, Biocatalysis in Organic Media, p. 279, Elsevier Sci. Publ., Amsterdam $(1987)$.

13. A. Geerlof, J.B.A. van Tol, J.A. Jongejan and J.A. Duine, I. Chrome, in press.

14. M.A. Bertola, H.S. Koper, G.T. Phillips, A.F. Merx and V.P. Claussen, Eur. Pat. Appl. 0244912A1.

15. P. van Ophem, J. van Beeumen and J.A. Duine, Eur. J, Biochem. 212, 819-826 (1993). 